\title{
Adulto Mayor: Participación e Identidad
}

\section{The Elderly Adult: Participation and Identity}

\author{
Hernán Zapata Farías *
}

\begin{abstract}
Resumen
El presente artículo hace una revisión de la conceptualización del término "Vejez" y las valoraciones asociadas al mismo concepto. Plantea que uno de los factores que influyen negativamente en la aceptación de esta etapa de vida denominada "Tercera Edad", y en la falta de reconocimiento de las personas en cuanto tal, es decir su pertenencia a este tramo etáreo, es un fenómeno relacionado con la difusión de la identidad asociado a la pérdida del rol social de este grupo. Se discute esta postura y se plantea la posibilidad de revertir este proceso incentivando la participación de los adultos mayores en redes de apoyo y a través del aporte que los otros, es decir los "no-viejos", pueden hacer en base al reconocimiento y validación del adulto mayor como un par. Se entiende que el sentido de indentidad personal, esto es "quien fui" ayer, "quien soy" hoy y "quien seré mañana" está conservado y que la identidad social es el componente de la función de identidad que se ve alterado.
\end{abstract}

Palabras Claves: Vejez, Adulto Mayor, Participación, Identidad

\section{Abstract}

In this article a review of the concept "Old age" and its significations are presented. It explains factors that involves accepting or rejecting this stage of life and analyses one of the most important factors related: That is, the lack of self acceptance of old aged people. In this article we put forward that this lack of acceptance starts a process of 'diffused identity'. This hypothesis is discussed and it makes the point that this process of diffusion of identity may be stopped by means of senior citizen participation and the achievement by old people of recognition by themselves and others as human persons with rigths and duties. Understanding that the meaning of personal identity - that is, that the one I was yesterday, the one I am now, and the one I will be tomorrow - would not be altered; what would indeed be modified is the social identity of senior citizens.

Key words: Old Age, Senior Citizen, Participation, Identity

\footnotetext{
* Psicólogo, Universidad de Chile, Master en Gestión Pública, Universidad de Barcelona Departamento de Desarrollo Institucional, hzapata@fosis.cl. (Trabajo presentado en el Congreso Mundial de la Federación Mundial de Salud Mental, Simposio Tercera Edad y Comunidad, realizado en Septiembre de 1999 en Santiago de Chile)
} 


\section{Sobre el Significado del Concepto "Vejez"}

De acuerdo al diccionario la palabra "vejez" se utiliza para referirse a la última etapa de la vida del hombre, por lo tanto sólo a una más dentro de las tantas que conforman el proceso de desarrollo vital. Sin embargo de por sí denota una mayor diferenciación y distancia del resto de la sociedad que la observada en otros estadios, cosa que no ocurre con la niñez o la juventud (Moraga, 1991). Suscita así reacciones negativas y no resulta sólo en una categoría descriptiva, sino que está cargada de significados que atribuyen los otros, no quienes viven el proceso es decir los propios viejos, sino que éstos, los viejos, se convierten en un otro para la sociedad y no en un otro cualquiera, sino que en un otro desconocido y más aún negado. Así, la palabra "Viejo" adquiere el valor de lo indeseado y es sinónimo de incapacidad o limitación.

Existe, afortunadamente, otra conceptualización de la vejez ya como etapa vital, la cual es más desconocida y se basa en el hecho evidente de que el paso del tiempo genera efectos en las personas, la cual ingresa a etapas distintas a las vividas previamente, constituyéndose cada una de ellas en una realidad propia y diferenciada, de manera similar a las de otras etapas ya vividas, es decir infancia, adolescencia o adultez.

Esta mirada adjudica a la vejez los potenciales propios de este estadio que la convierten en única: serenidad de juicio, experiencia, conocimiento, madurez vital y perspectiva de la historia personal y social. $\mathrm{Cu}$ riosamente estas potencialidades antaño enormemente valoradas hoy no lo son tanto y han llevado al adulto mayor a la paradoja del rol sin rol y a la consecuencia más grave desde el punto de vista psicológico, la de la difusión de la identidad o como dice Erickson la desesperanza versus la integridad, inte- gridad que sería el curso natural del desarrollo si es que el medio proveyera las condiciones adecuadas.

\section{Sobre el Desarrollo del Proceso de Identidad}

En nuestra sociedad la consolidación de la identidad está asociada a la asunción de las tareas adultas y específicamente al ingreso al mundo del trabajo. Su consolidación favorece la definición de los límites, tanto individuales como sociales, el sentido de pertenencia y la adjudicación de responsabilidades. "La identidad sirve para organizar la interpretación de las experiencias asignándoles un significado subjetivo que puede ser modificado por la propia experiencia" (Moraga, 1991).

Desafortunadamente en el caso del adulto mayor esta capacidad, proveniente de la consolidación de la identidad, es la que se ve mayormente afectada, ya que la persona mayor pierde el principal referente de identificación que generalmente es el rol que entrega el puesto laboral o las tareas propias del adulto. Dicho aspecto para las personas mayores ya no existe puesto que se supone que no debe ejercerlas. En otras palabras, en la práctica, se concede al adulto mayor un dudoso privilegio: el derecho a hacer nada.

Desde este punto de vista la Sociedad ha cometido una falla inexcusable en cuanto a su capacidad para definir un conjunto de actividades significativas específicas de las personas mayores que ayudara a ganar el reconocimiento social (Barros, 1999). "La identidad social se levanta como una construcción representativa de sí en relación en relación con los otros y la sociedad "(Moscovici, 1981). La "Identidad" entendida como proceso puede avanzar hacia la consolidación o la difusión. La consolidación es también un proceso que puede involucionar lo que la diferencia de un estado. Si bien el reconocimiento del yo indivi- 
dual, es decir quien soy hoy, quien fui ayer y quien seré mañana está mantenido; la función de la identidad social se ve alterada y por lo tanto lo que plantea Maritza Montero (1993) en relación a que la identidad social “ ..proporciona un marco de referencia dando a la definición individual un sentido y proyección histórico .." se ve interferido.

Freud ya decía que para la infancia las tareas prescritas están referidas a las actividades lúdicas y al estudio, para la adultez los roles definidos en la familia y en el ambiente laboral y, para el adulto mayor, ... todo esto se desconoce. Probablemente si Freud viviera hoy su juicio sería distinto o por lo menos un intento de respuesta ya habría sido propuesto, dado que en su época la expectativa de vida no superaba los 45 años. Sin embargo no es posible cerrar los ojos en cuanto a lo poco que se ha avanzado respecto de este tema desde principios de siglo.

\section{La Negación de la "Vejez" como Etapa Natural de la Vida}

Hoy, más que intentar profundizar en el tema, éste es más bien negado del mismo modo que se niega la muerte y la asociación que se produce entre la vejez y el morir, ayudan a mantener esta mirada de desconocimiento que inunda nuestra sociedad. Es necesario conocer a ese otro que es el viejo para establecer el diálogo, conocerlo en su identidad y en su ser persona con capacidad para decidir sobre los distintos aspectos que conforman su diario vivir.

Esta negación ha generado una consecuencia de suyo negativa, la incapacidad del adulto mayor para reconocerse a sí mismo en cuanto tal y más aún, esta negación ha llevado al adulto mayor a identificarse en torno a parcialidades en torno a, por ejemplo, el ser diabético, hipertenso u otra característica de este tipo, en que evidentemente la persona al estar asumida sólo parcialmente no logra identificarse ni sentirse integrada.

Es cierto que algunos autores discuten este aspecto mencionando que esta desventaja es propia de los hombres y no de las mujeres. Los hombres, continúan los mismos autores, no solo han logrado su sentido de pertenencia a través del espacio laboral sino que además sus redes de apoyo las han estructurado a partir del rol de hombre-trabajador. Se plantea que para las mujeres esta desventaja es menor puesto que tienen mayor entrenamiento en mantener redes de apoyo fuera del espacio laboral. No desconocemos este hecho pero existe evidente pérdida del rol adulto aún en las mujeres, pues incluso la labor asociada a los aspectos familiares se ve afectada y ya no se desarrolla en ese espacio con las mismas características que antes y si bien su nivel de participación en distintas redes es relativamente alta, sistemáticamente ésta se ve reducida a medida que avanzan los años.

\section{Adulto Mayor y Participación}

Existen opiniones que coinciden en que la participación en organizaciones comunitarias permite al adulto mayor canalizar adecuadamente su tiempo libre y al mismo tiempo contribuir a mejorar su calidad de vida. Es más, la actividad comunitaria es una opción válida para aportar soluciones de adultos mayores para los adultos mayores (Romo, Ma.1999). Esta forma de integración permite al adulto mayor contactarse con su grupo de pares y compartir más allá de la carencia, desarrollando potencialidades a partir de este contacto.

Todos llegamos a ser viejos, sin embargo la manera como envejecemos y la calidad de nuestras vidas en la vejez se relaciona con los 
recursos intelectuales, sociales, biológicos y materiales acumulados durante el ciclo vital. Parte importante de las características asociadas a la vejez parecen responder en gran medida a un proceso de construcción social y por lo tanto los mitos creados en torno a ésta son los que han contribuido sustancialmente a separar a los viejos del resto de la sociedad, responsabilidad especialmente del mito de la pasividad asociado a esta edad. Actualmente una persona adulta mayor de 60 años tiene aún todo un horizonte por delante y la sociedad no se ha preparado para brindar el espacio acogedor que legítimamente les pertenece y no deberían estar reclamando. Contribuir de esta manera a mejorar la calidad de vida es tarea ineludible.

\section{Sobre el Concepto de "Calidad de Vida"}

El término "Calidad de Vida" aparece en la década del 70 y tuvo su máxima expansión hacia los años 80 , encubierto por el desarrollo de conceptos como los de bienestar, salud y felicidad. Algunos plantean que su desarrollo inicial se originó en las ciencias médicas para extenderse rápidamente a la psicología y la sociología, en el afán de desplazar otros términos de difícil operacionalización como bienestar y felicidad (Moreno, 1996).

La calidad de vida aparece como la resultante de interacción de áreas objetivas y subjetivas y es incapaz de ser evaluada a partir de uno de estos dos polos o focos. El papel de los factores psicológicos como condicionantes del grado de satisfacción o bienestar es determinante para este proceso. Desde la Psicología, la calidad de vida se convierte en un concepto referido básicamente al tipo de respuesta individual y social ante el conjunto de situaciones reales de la vida diaria, y en este sentido más que una defini- ción teórica existe acuerdo entre los investigadores en relación a las dimensiones componentes de la calidad de vida, considerándolo como un constructo incluyente de todos los factores que impactan sobre la vida del individuo (Grau, 1996).

Es reconocido que uno de los factores que mayormente influyen sobre la calidad de vida del adulto mayor es el tema del aislamiento y abandono (FOSIS, 1996), o dicho de otra forma la desconexión con las redes de apoyo social.

\section{Las redes de Apoyo Social y el Adulto Mayor}

La red social está constituida por todas aquellas relaciones significativas que una persona establece cotidianamente y a lo largo de su vida. En ese sentido cada persona es el centro de su propia red, que está formada por sus familiares más cercanos, los amigos, compañeros de estudio y de trabajo, los miembros de las instituciones a las que acuden habitualmente y los conocidos con quienes se ve sólo esporádicamente.

Además de las dimensiones estructurales mencionadas, las redes sociales pueden caracterizarse en relación a las funciones que cumplen. Las más importantes se refieren a la compañía social, apoyo emocional, guía y consejo e intercambio de información, regulación social y apoyo instrumental. La red social personal cumple funciones importantes para el bienestar psico-social del individuo, como es la de posibilitar la inserción social de la persona, y es básico para el desarrollo de su identidad, por lo tanto no sólo es relevante en situaciones de crisis. Cada punto de la red es irreemplazable y es a la vez depositaria de la historia personal, de una parte de la propia identidad, de la retroalimentación social y del cuidado de la salud.

El apoyo social que otorgan las redes ha demostrado ser un factor muy importante al 
evaluar el impacto que las crisis de vida tienen en las personas. Por otra parte las personas que cuentan con redes sociales activas tienen menor probabilidad de deprimirse luego de pérdidas importantes (Gottlieb; 1996)

Un primer nivel de la participación se da en este terreno, en el acceso a la red social y a través de éstas a las organizaciones comunitarias, sin embargo no es la única forma ni es la única instancia de participación, también se trata de generar ciudadanía es decir personas con derechos y deberes. Según el informe de desarrollo humano elaborado por el Programa de las Naciones Unidas para el Desarrollo, PNUD, Chile tiene el más alto índice de desarrollo humano en América Latina y esto es necesario que se vea reflejado para el caso del adulto mayor.

La participación de la población adulta mayor se ha caracterizado históricamente por ser más bien pasiva, marcada por la recepción de acciones más que por la gestión de sus propias tareas, lo que la sitúa más cerca de la marginación social que de la participación, si es que entendemos ésta como un proceso capaz de satisfacer necesidades desde la definición de sus propios problemas y también como la capacidad para tomar decisiones en pos de la solución de los mismos.

Los distintos enfoques sobre el envejecimiento, que como ya dijimos son planteados desde otros y no desde la realidad del propio adulto mayor, han influido fuertemente en la forma en que las personas mayores se han asociado.

La perspectiva de una vejez desvalida ha dado origen a instituciones orientadas a dar ayuda a los adultos mayores desde una postura básicamente asistencial, organizada para atender a los ancianos haciéndose cargo de necesidades básicas de subsistencia, esperando muy poco de los propios adultos mayores.

Desde el enfoque clásico del Estado se ha planteado que las personas mayores ya han hecho su aporte a la sociedad, por lo tanto éste se compromete a retribuir medios de subsistencia, respondiendo a la ideología de la Seguridad Social, generando un tipo de asociación básicamente reivindicativa.

El desafío que queda por asumir por parte de la sociedad, es de entender y aceptar la vejez como una etapa más de la vida que tiene como norte la integridad y el desapego, situación que es sólo posible si un medio amigable ha entregado las posibilidades de que así sea. Jung plantea que durante esta etapa se produce una natural introspección y "las relaciones, la comprensión del significado de la vida y el deseo de darnos a los demás se convierte en nuestras preocupaciones" (Jung, citado por Belsky, 1996).

La no aceptación de estas tendencias naturales y el quedarnos aún en el período de la adultez joven lleva a la Persona Mayor a "estancarse, tener la sensación de inutilidad, sentirse desgraciados y rígidos" ((Jung, citado por Belsky, 1996).. Sin embargo si el desarrollo procede de una manera ideal se alcanza la culminación, "podemos transformarnos en un ser espiritual" (Jung, citado por Belsky, 1996)., trascender y alcanzar el desapego.

Si se entiende así esta etapa, es posible que surjan instituciones capaces de poner énfasis en el potencial de las personas mayores para su propio desarrollo, transformando ésta en una fuerza social que genera asociaciones de adultos mayores con capacidad para interlocutar ante las autoridades y ante la sociedad civil por sus propios derechos.

En el sector gubernamental se han realizado esfuerzos programáticos en las áreas de salud, recreación, turismo y desarrollo social, favorecedoras de la generación de grupos cuyo éxito en la organización independiente se relaciona con la anticipación de las consecuencias de la oferta de los servicios públicos. Por otra parte las municipalidades a través de la 
Oficina del Adulto Mayor han intentado también generar agrupaciones autónomas, entregando básicamente servicios culturales, recreacionales y asistenciales, en base a una estrategia de asociación generada por frecuencia de encuentro.

La Iglesia Católica a partir de 1968 y a través de Caritas, desarrolla una estrategia destinada a la creación de clubes parroquiales a través de la Federación de Clubes de Ancianos. En 1982 se descentraliza la Federación de Clubes que pasa a depender de las Vicarías, lográndose "diversidad en las ofertas y un mayor grado de participación local" (Mideplan, 1990).

En 1984 se realizó un Encuentro Nacional de la Tercera Edad, llamado "Los Ancianos en Chile hablan", a fin de que los propios adultos mayores se pronunciaren sobre el Plan Internacional de Viena, sin embargo actualmente la acción de la Iglesia Católica ha puesto mayor énfasis en los aspectos pastorales que en la generación de autonomía y se ha suscitado una serie de pequeños desencuentros con el programa "Más Vida para tus Años" del Fondo de Solidaridad e Inversión Social, FOSIS, el cual exige a los grupos de adultos mayores, en la perspectiva de generar autonomía, personería jurídica con lo cual no están de acuerdo todos los vicariatos.

CONAPRAN, Consejo Nacional de Protección a la Ancianidad, se crea el año 1974 presidido por la esposa del Comandante en Jefe de la Fuerza Aérea de Chile. Inicialmente realiza una labor predominantemente coordinadora, para después realizar labor asistencial casi exclusivamente. En los últimos años su labor ha estado dirigida a coordinarse con los sectores correspondientes de manera que sus iniciativas de servicios sean realizadas en conjunto con éstos.

El sector privado sin fines de lucro, ONG's y otros, han realizado también un gran esfuerzo dirigido a fomentar la asociatividad del adulto mayor, especialmente a través de la Red de Programas para Adultos Mayores.

Hasta hoy las modalidades de agrupación que convocan a los adultos mayores pueden caracterizarse en:

1. Hogares de Ancianos, los cuales brindan alimentación, habitación y cuidado con nula acción de los propios adultos mayores.

2. Los Clubes de Adultos Mayores, que buscan promover el intercambio de relaciones, estimular expresiones de apoyo y de solidaridad

3. Las Asociaciones de Pensionados, que se forman en defensa de los derechos adquiridos y reivindican su historia.

En otro nivel de acción se ubica la Coordinadora Nacional de adultos mayores, con apoyo del Ministerio Secretaría General de Gobierno, que busca constituirse en una instancia de interlocución válida, con resultados que hoy distan de haber logrado este propósito.

Las Naciones Unidas han planteado, a partir de la reunión de Montevideo del año 1997, la idea fuerza de una "Sociedad para todas las Edades", lo cual se complementa con otras ideas surgidas en el plano nacional como la de "Favorecer la integración social del Adulto Mayor a través del fortalecimiento de sus organizaciones" (Comité Nacional para el Adulto Mayor, 1996).

\section{A modo de Conclusiones}

Como ya dijimos la desconexión de las redes de apoyo y la escasa participación en la organización social, atentan contra la integración y al atentar contra la integración atentan también contra la identidad. La ta- 
rea que queda por realizar se relaciona con este aspecto, es decir que el adulto mayor se reconozca a sí mismo como tal y no sólo en torno a parcialidades. La aceptación por parte de la sociedad de la vejez como una etapa más del desarrollo es fundamental para este esfuerzo. Se dice, connotándolo como una característica de desventaja, que el adulto mayor vive en el pasado, fenómeno que ciertamente es así, pero que no necesariamente tiene una significación negativa.

Cierto es que vivenciar desde el pasado está cerca de la posición depresiva, pero también sabemos que la depresión como entidad clínica no tiene una prevalencia más alta en los adultos mayores que en otras épocas de la vida, es más, la prevalencia de depresión es mayor en el grupo de 30 a 54 años (Rojas, G. 1999); por lo tanto ese vivir en el pasado tiene también el valor de rescate de la cultura y del saber.

Esta gran capacidad del adulto mayor, del mismo modo que la capacidad de entregar afecto, no han sido valoradas en su justa dimensión y esa falta de reconocimiento ha contribuido también a la desintegración. Como dicen Antonucci y Jackson "las conductas de apoyo social proporcionan verdadero apoyo sólo si influyen positivamente en la eficacia personal del receptor de este apoyo en las conductas cotidianas" ( Antonucci y Jackson, 1990).

El creer y percibir al otro como persona con capacidades influye positivamente en la autopercepción, por ende en la autoestima y en la función de identidad.

Este proceso se grafica en la Figura 1, que se muestra en el siguiente cuadro:

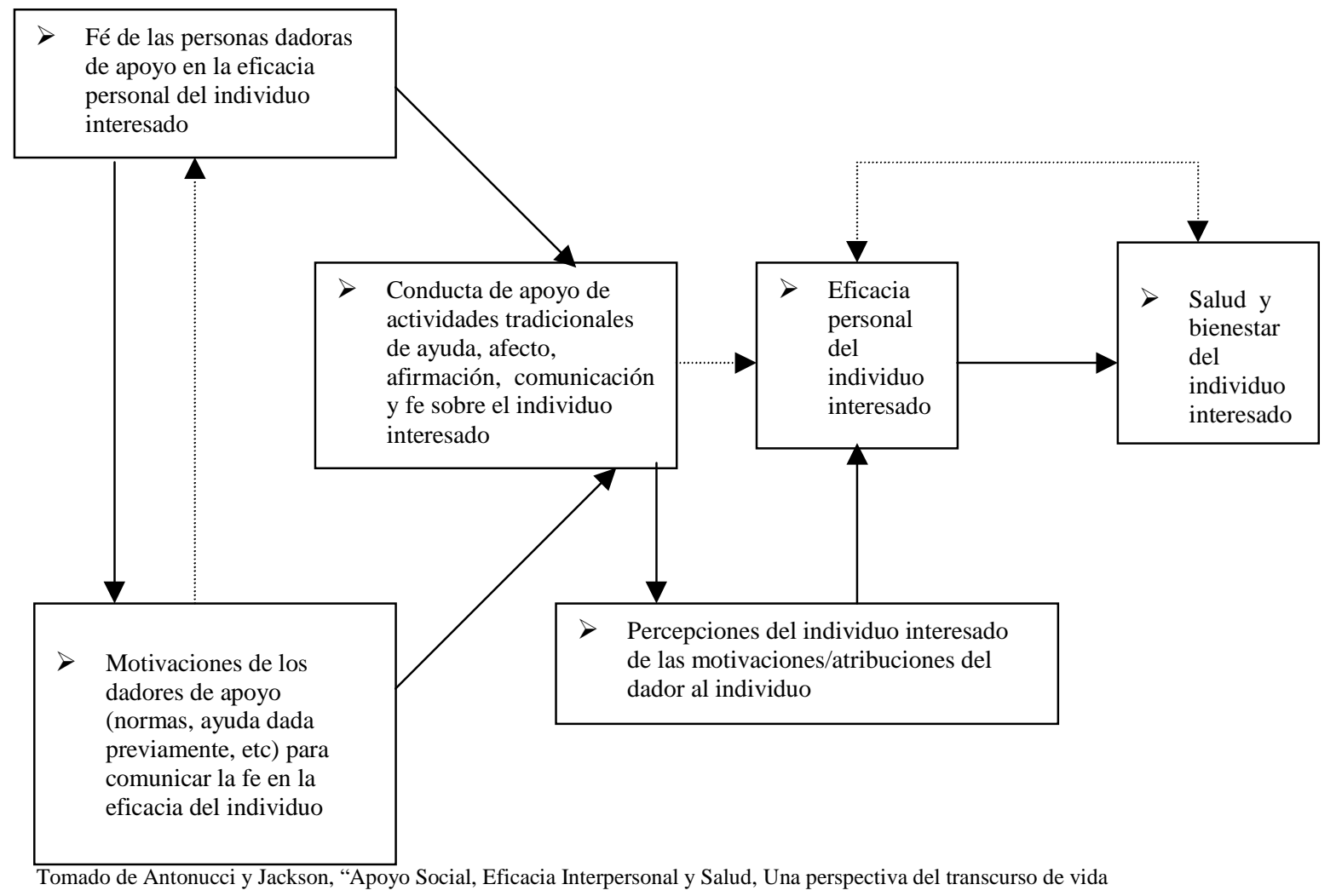


Como vemos la participación tiene sentido en la medida que la relación que se establece es entre personas con derechos y deberes, en una interacción en que ambos tienen fe en la capacidad del otro para ser reconocido como tal y en la capacidad de cada uno, es decir desde la autopercepción de la eficacia personal.

Esta valoración positiva es la que influirá positivamente en la función de identidad, convirtiéndose por lo tanto la participación y la relación con otros en motor fundamen- tal de la integración. Evidentemente este circuito como lo plantean sus autores puede tener un efecto negativo, que es lo que ha ocurrido hasta ahora en que se ha retroalimentado la percepción de los adultos mayores como pasivos y la entrega de conductas de apoyo como poco sinceras. La disposición del dador de apoyo para practicar la conducta de apoyo es clave reconociendo la autoeficacia del adulto mayor.

Propongo el siguiente esquema, modificado de la Figura 1 y que llamaremos Figura 2, para graficar este reconocimiento.

Figura 2: Percepción de Autoeficacia e Identidad:

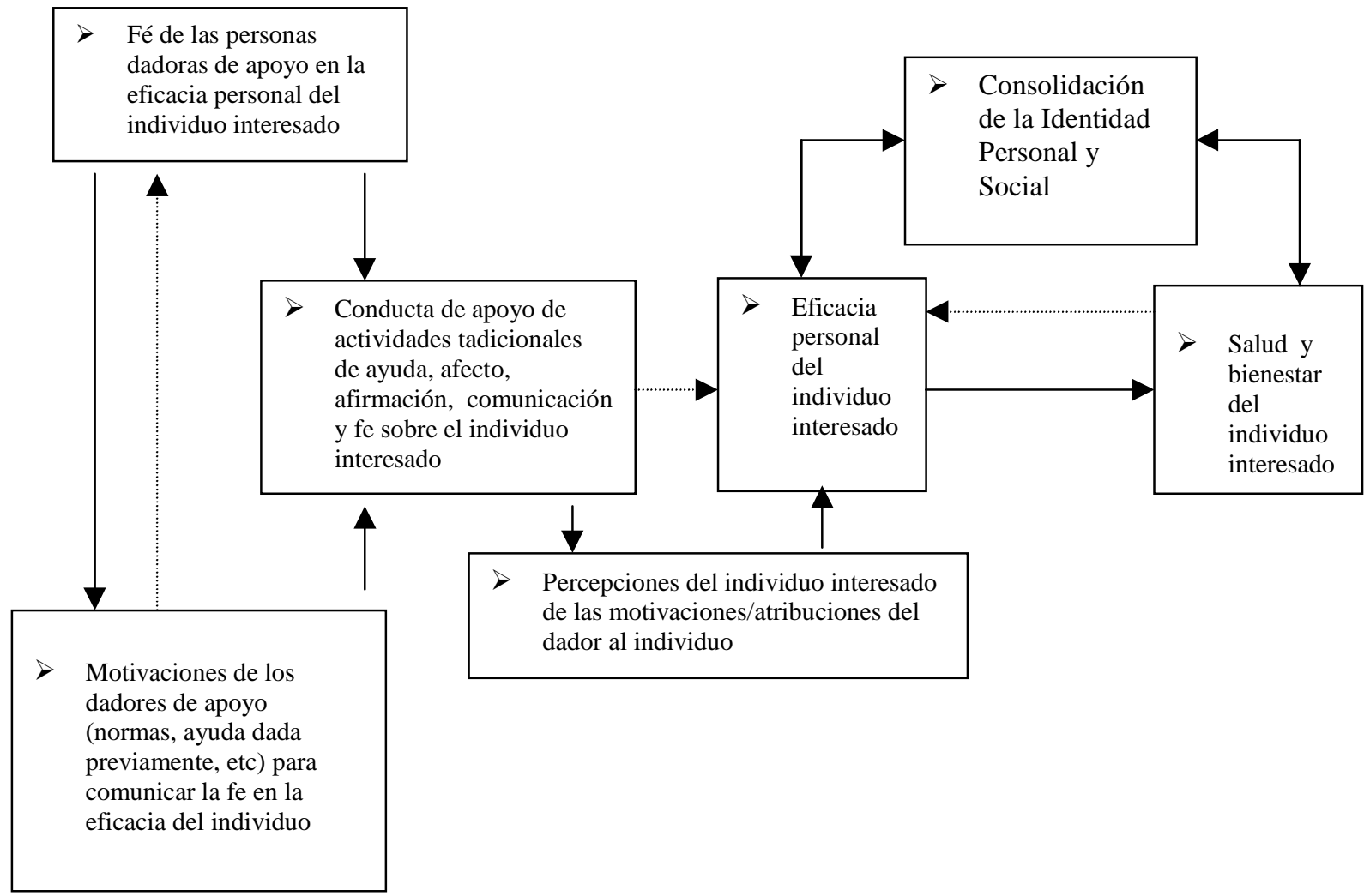

Modificado de Antonucci y Jackson, “Apoyo Social, Eficacia Interpersonal y Salud, Una perspectiva del transcurso de vida 
Finalmente es necesario recalcar que la participación no se agota ni en la red social ni en la organización comunitaria, participación es también que los adultos mayores tengan el derecho a definir que tipo de acciones se requiere realizar para mejorar su calidad de vida.

Las acciones de apoyo deben considerar el favorecer y fomentar el desarrollo de las potencialidades del Adulto Mayor instalando capacidades en los grupos y personas, generando entre las organizaciones relaciones de cooperación, encuentro y asociación.

Cómo dice Hermann Hesse "La vejez es una etapa de nuestra vida y, como todas las otras, tiene su propio rostro, una atmósfera y una temperatura propias, su alegría y sus penas".

\section{Bibliografía}

1. Antonucci, T; Jackson, J; Apoyo social, eficacia interpersonal y Salud: Una perspectiva del transcurso de la vida, 1990

2. Barros, C." El adulto mayor en la sociedad chilena", Gerontología Básica, Lecturas Complementarias, P.U.C., Teleduc, 1999

3. Comité Nacional para el Adulto Mayor, 1996, Gobierno de Chile.

4. FOSIS, Programa del adulto mayor “Más Vida para tus Años”, 1996
5. Gottlieb, B., citado en "Análisis de una intervención familiar comunitaria en violencia intrafamiliar" Villela y otros, Revista Chilena de Psicología, Vol. 17, Vol. 18, 19961997

6. Grau, J.: Calidad de Vida y Salud: Problemas actuales en su investigación, . Revista Chilena de Psicología, Vol. 17, Vol. 18, 1996-1997

7. Jung, citado en "Psicología del Enevejecimiento", Belsky, J; 1996, pág.27.

8. Mideplan, Envejecimiento en Chile

9. Montero, M., Ideología, Identidad Social, Ideología y Transformación en América Latina, Conferencia UMCE, Santiago, 1991,

10. Moragas, R., Gerontología Social, Ed. Herder, 1991.

11. Moreno, Jiménez, 1996, "evaluación de la calidad de vida" citado en "Calidad de Vida y Salud: problemas actuales en su investigación" de Grau, J. Revista Chilena de Psicología, Vol. 17, Vol. 18, 1996-1997

12. Moscovicci, $S$, Introducción a la Psicología Social, Paidós, Buenos Aires, 1981

13. Rojas, Graciela. VI Congreso de Internacional de Geriatría y Gerontología de Chile, 1999

14. Romo, Ma. Elena, "Arte y Recreación en el adulto mayor" en Seminario "Aprendiendo a Envejecer", U. De Chile 
